\title{
Screening for and Surveillance of Barrett's Esophagus Is Clinically Indicated
}

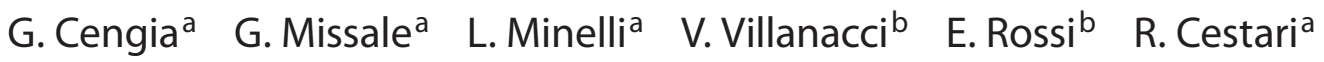 \\ ${ }^{a}$ Department of Surgery and ${ }^{b}$ Second Department of Pathology, Digestive Endoscopy, University of Brescia, \\ Spedali Civili, Brescia, Italy
}

\section{Key Words}

Barrett's esophagus • Barrett's esophagus, screening and surveillance - Esophageal adenocarcinoma $\cdot$ High-grade dysplasia $\cdot$ Intestinal metaplasia

\begin{abstract}
Barrett's esophagus (BE) is a complication of chronic gastroesophageal reflux disease (GERD) and is the precursor of esophageal adenocarcinoma (EA), through a progression from intestinal metaplasia (IM), through high-grade dysplasia (HGD). Although the progression from BE to EA seems to be infrequent $(0.5 \%$ per year), endoscopic and bioptic surveillance would play a significant role in the evaluation of HGD and the detection of EA in early, curable stage, improving survival rates after treatments. The severity and the duration of GERD could be helpful in the assessment of the risk for $B E$ and to enroll these subjects into screening protocols to detect any dysplastic or neoplastic change. The benefits of screening-surveillance programs could be furthermore enhanced by an improvement in diagnostic methods, such as high-resolution endoscopic techniques and the use of biomarkers for the histological examination seems to play a primary role in the cancer risk stratification; in such way, endoscopic resection techniques (mucosal resection and submucosal dissection) can be considered as a helpful method to stage dysplastic changes in BE.
\end{abstract}

Copyright $\odot 2007$ S. Karger AG, Basel
(C) 2007 S. Karger AG, Basel

0257-2753/07/0253-0197\$23.50/0

Fax +4161306 1234

E-Mail karger@karger.ch

www.karger.com
Accessible online at: www.karger.com/ddi

\section{Introduction}

Barrett's esophagus (BE) is considered the major risk factor for the development of esophageal adenocarcinoma (EA) (30-125 times higher than in the general population), even if the incidence of this cancer in the BE population ranges extremely, and has been confirmed in many prospective studies and also recently by Conio et al. [1] who reported cancer in 1/220 surveyed BE patientyears at follow-up $(0.45 \%)$. At the time of diagnosis, up to $50 \%$ of patients have an advanced cancer with a poor prognosis and overall 5 -year survival rate $<10 \%$ after surgical resection [2]. BE affects approximately $6-12 \%$ of patients symptomatic for gastroesophageal reflux disease (GERD), $1 \%$ or less of unselected subjects and in $10-25 \%$ of asymptomatic individuals undergoing endoscopic examination $[3,4]$.

In the natural course of $\mathrm{BE}$, many controversies still exist regarding the likelihood to progress to cancer and the optimal management to prevent the onset of EA; nevertheless, the risk for the development of cancer is related to a well-defined sequence from intestinal metaplasia (IM), through low-grade (LGD) and high-grade dysplasia (HGD) [5]. Several changes in gene structure, gene expression and protein structures are involved in this multistep process, consisting in the initiation phase affecting genetically predisposed individuals exposed to chronic

Prof. Renzo Cestari

Department of Surgery, Digestive Endoscopy

University of Brescia, Spedali Civili di Brescia

Piazzale Spedali Civili 1, IT-25125 Brescia (Italy)

Tel. +390303995 539, E-Mail cestari@med.unibs.it 
Table 1. American College of Gastroenterology Guidelines for BE surveillance intervals [adapted from 11]

\begin{tabular}{|c|c|c|}
\hline Dysplasia & Documentation & Follow-up endoscopy \\
\hline None & 2 endoscopies with biopsy & 3 years \\
\hline Low-grade & Highest grade on repeat & 1 year until no dysplasia \\
\hline \multirow[t]{3}{*}{ High-grade } & Repeat endoscopy with biopsy to exclude cancer & Focal - every 3 months \\
\hline & and document high-grade dysplasia & Multifocal - intervention \\
\hline & Confirm with expert pathologist & Mucosal irregularity - endoscopic mucosal resection \\
\hline
\end{tabular}

reflux which leads to the formation of columnar-lined epithelium (CLE) with IM; the formation phase with the enlargement of this metaplastic tissue and the progression phase characterized by the onset of histological changes leading to dysplasia which is therefore the best marker of potential malignancy.

\section{Screening and Surveillance}

$\mathrm{BE}$, suspected endoscopically when CLE is found in the distal esophagus, must be confirmed by the detection of IM on histology, defined by the presence of goblet cells. In order to achieve benefits from screening patients for $\mathrm{BE}$, the knowledge of its real prevalence in the general population is a primary endpoint, as recently reported in asymptomatic subjects [4] and in non-GERD patients, suggesting that $\mathrm{BE}$ can be unrecognized until the endoscopy has been performed and its prevalence may be higher than presumed [6].

Since BE is a well-known complication of GERD, international guidelines suggest to perform upper endoscopy in patients with chronic reflux symptoms, detecting $\mathrm{BE}$ in approximately $10-15 \%$ [3]; therefore, the frequency (daily) and the duration (from 5 up to 10 years) of reflux symptoms are considered risk factors for EA, with an odds ratio of 7.7 (95\% CI 5.3-11.4) and 3.0-6.4 (p 0.001), as reported by Lagergren et al. [7]. Similarly, other factors such as age $>50$ years, male sex, and Caucasian race have also been shown to be associated with a risk of developing $\mathrm{BE}$ and EA. These data support the 'once-a-life endoscopy' as a cost-effective strategy only in a selected, highrisk population, as proposed by Inadomi et al. [8], who demonstrated the potential benefits of screening and surveillance for $\mathrm{BE}$ and related cancer among white, symptomatic males at the age of 50 and limiting the surveillance to patients with dysplastic findings with an incremental cost-effectiveness ratio compared to no screening or surveillance.
Endoscopic surveillance in the BE population should therefore be performed to detect dysplasia and early stage cancer, which can lead to a significant improvement in the survival rate after surgery. Several retrospective studies have shown that cancers detected during the surveillance period are more likely to have an early stage, compared with those detected outside the program. Van Sandick et al. [9] demonstrated only 1 case of EA (6\%) with nodal involvement in the surveyed population compared with $34(63 \%)$ in the non-surveyed group ( $p<$ $0.0001)$, with a significant better survival rate (85.9 vs. $43.3 \%$ at 2 years). Moreover, in the population study from Corley et al. [10] among 23 patients with BE diagnosed $>6$ months before evidence of cancer, $75 \%$ of cancers $(\mathrm{n}=15)$ detected by surveillance were alive at the end of follow-up, compared with no one in the non-surveyed group of cancers $(n=8)(p<0.001)$. These results strongly suggest the primary role of surveillance in detecting early-stage cancers suitable for curative treatment.

Currently, the optimal surveillance strategy seems to be the one proposed and updated by the American College of Gastroenterologists in 1999 and 2002 (table 1), even in the absence of randomized controlled studies which assess the cost-effectiveness of these programs [11]. The diagnosis of dysplasia is indeed the crucial point in the management of $\mathrm{BE}$, since the patient might progress to cancer; therefore, the histological confirmation requires an experienced pathologist and a standardized fashion (correct orientation). To reach the correct analysis, 'transient dysplasia', defined as not confirmed in a subsequent specimen, must be ruled out, ranging from 28 up to $61 \%$ in surveillance protocols for BE, and it seems to be related to sampling errors, associated inflammatory changes or interobserver variations $[12,13]$. Furthermore, the grading of dysplasia can be difficult due to the high interobserver variability among pathologists and to the distribution of dysplastic abnormalities in esophageal mucosa, leading to sampling errors during the bioptic protocols. For the assessment of the dysplastic pattern it 
is fundamental to choose the appropriate management: dysplasia can be classified into unifocal (HGD found in $<5$ crypts in a single bioptic specimen), multifocal ('flat type') or associated with 'visible lesions' ('non-flat type'). The presence of multifocal HGD is correlated with a fourfold higher risk of cancer development (55\%), compared with focal changes (15\%) [14], and the presence of nodularity is frequently associated with underlying or associated cancer. These results showed that patients with focal HGD survived longer than patients with diffuse HGD [15]. Furthermore, HGD is still controversial in clinical practice, especially regarding the options for the treatment, such intensive surveillance, endoscopic mucosal resection or surgery which are due to the extremely wide variability in the progression to cancer.

Retrospective studies from Schnell et al. [16] after a 7.3-year follow-up demonstrated no cancer progression from HGD in 63 of 79 cases, suggesting that an intensive surveillance program could be employed in such highrisk patients. Recently the results of 'the hunt and surveillance protocol' identified cancer in 12 patients out of 75 with flat HGD who underwent surveillance after the hunt (16\%), avoiding unnecessary esophagectomy in the majority of surveyed subjects; moreover, among the cancers discovered during the protocol, 9 were in stage 1, 1 in stage 2, 1 died from unrelated causes and 1 dropped out. This 26-year study provided strong evidence that the HGD in BE will not progress to cancer in the majority of enrolled subjects (85\%); therefore, surgery can be safely avoided. Furthermore, in the $15 \%$ of cases in which cancer will eventually be detected, the surgical resection will be curative, due to the early stage of the disease. An intensive surveillance program, scheduled at 6- to 12month intervals, therefore seems to be able to detect all potential cancers arising from $\mathrm{BE}$ at an early curable stage [17].

The cost-effectiveness of screening and surveillance programs could be improved by identification of clinical, endoscopic and histological features, and easy employment that can accurately assess the risk for neoplasia (risk stratification) and provide criteria to optimize the management of BE. BE length has been identified as an important factor for the onset of dysplasia and cancer risk progression, as reported by Hirota et al. [18], who found significant differences in dysplasia and cancer prevalence in patients with long-segment BE (LSBE) (31\%) compared to short-segment BE (SSBE) (10\%) and to cardiac metaplasia (6.4\%). Similarly, Weston et al. [19] demonstrated a prevalence of dysplasia at $24.1 \%$ for LSBE, compared to $8.1 \%$ in SSBE $(\mathrm{p}<0.0001)$; furthermore, prevalence of

Screening for and Surveillance of BE Is

Clinically Indicated cancer was significantly higher in LSBE (15.4\%) than in SSBE $(0 \%)(p<0.005)$.

Baseline dysplasia is a good predictive factor for progression to cancer, as reported by Dulai et al. [20] (1.28\% per year; $\mathrm{p}=0.001)$ compared with no dysplastic changes at baseline endoscopy $(0.36 \%$ per year). Moreover, HGD has been well defined as a primary marker for the progression to neoplasia in BE epithelium, with an extremely wide variation rate ranging from 33 to $55 \%$.

\section{Endoscopic Diagnosis}

One of the limitations of the current surveillance practice is the poor capability to perform a complete histological analysis of the dysplastic tissues with the standard bioptic protocols, with the risk of understaging, since HGD can be frequently associated with intraepithelial focal cancer ( $>40 \%$ of surgical specimens) and the presence of cancer ranges from 7 to $14 \%$ in focal HGD to $38 \%$ up to $56 \%$ in diffuse (multifocal) HGD. The risk on lymph node metastases increases with the depth of neoplastic invasion, up to rapid invasion of the submucosal layer; therefore, more accurate diagnostic methods would be needed in order to improve the assessment of the dysplastic tissue and to stage the neoplastic mural invasion [21].

The main limitation of routine videoendoscopy is the need for biopsy and histologic evaluation to distinguish IM from fundic or cardiac epithelium and the difficulties in the detection and grading of dysplasia. Additional techniques should therefore be used to improve endoscopic performance in patients suspected for BE or dysplasia, to minimize the risk of sampling errors. Endoscopes with high-resolution charged coupled device (CCD) chips provide a superior image quality and show the superior ability to discriminate details in the nonmagnified overview image. Such an instrument is the key requirement of a screening tool: the detection capability provides an even more detailed image by optically enlarging the mucosal surface area.

Endoscopic staining techniques (chromoendoscopy) using absorptive and contrast stains can be used to enhance the visual characteristics of both normal and suspected abnormal tissue creating a visual distinction for early detection, targeted biopsy and treatment. These data, confirmed by Kiesslich et al. [22], provided histological evidence of IM with a sensitivity of $98 \%$ and a specificity of $61 \%$ among targeted biopsies of MB-stained areas. Recently, a multicenter study from Sharma et al. 
[23] showed magnification chromoendoscopy able to identify IM and dysplasia or cancer in BE through the capability of target biopsies on selected areas according to three pathological mucosal patterns (ridge/villous, circular and irregular/distorted) sensitive and specific for HGD and cancer.

Narrow band imaging (NBI) is a new technique that uses optical filters for the imaging of the mucosal morphology, consisting of both mucosal and vascular patterns without the use of dye spraying. NBI is based on the phenomenon that the depth of light penetration depends on its wavelength; the longer the wavelength, the deeper the penetration. Blue light penetrates, therefore, only superficially, whereas red light penetrates into deeper layers. In NBI, RGB (red, green, blue) filters with narrow bandpass ranges and a higher relative intensity of blue light are used. These filters enable better visualization of the mucosal patterns, because blue light allows for optimal superficial imaging. In addition, NBI reveals the superficial vasculature because of absorption of the blue light by hemoglobin [24]. The detection of the mucosal pattern should be followed by the analysis of any irregularity, even in the vascular pattern, such as abnormal vessel formation, predicting IM or HGIN [25]. Recent results from Fock et al. [26] confirmed the value of NBI in BE detection among chronic GERD subjects (40.6\%) showing a villous pattern confirmed by histology in $60.9 \%$ of the specimens [27].

Similarly, Fuji Intelligent Chromoendoscopy (FICE) is a new promising high-definition electronic chromoendoscopic technique for the enhancement of vascular images, which allows to detect arranged abnormal vessels such as in cancerous lesions.

Light-induced fluorescence spectroscopy may be used to evaluate the malignant or benign presentation of $\mathrm{BE}$, LGD and HGD based on the assumption that different fluorescence patterns will be seen in these conditions compared with normal mucosa. Light-scattering spectroscopy, a technique using white light from fiberoptics during endoscopy, has been used to detect dysplastic nuclei crowding and enlargement. High levels of specificity and sensitivity in identifying LGD and HGD have been reported. Recently, unsedated upper endoscopy using small-caliber instruments has gained interest as a potential diagnostic tool in the screening procedure because of its feasibility, acceptability and accurateness when compared with conventional sedated endoscopy [28]; this may lead to potential advantages in the reduction of sedation-related complications and costs, and seems to be more cost-effective than the standard procedure; this would make both screening and surveillance endoscopy more proposable. However, limited information is available on unsedated endoscopy in the evaluation of BE, and there are no studies examining its utility in screening. Although there is little evidence comparing unsedated small-caliber endoscopy with conventional endoscopy for the diagnosis of $\mathrm{BE}$, the statement that this technique was an acceptable alternative approach for the screening of BE has recently been accepted [3].

Endoscopic mucosal resection (EMR) seems to be the best technique to obtain a large sample, achieving the most accurate staging through the evaluation of mucosal and submucosal layer, 'en bloc', leading to minimize interobserver variability. Moreover, EMR can be considered an excellent staging technique, compared to endoscopic biopsy and high-frequency ultrasonography in the assessment of mucosal versus submucosal cancer invasion, with a diagnostic gain of up to $40 \%$ compared to standard bioptic techniques [29]. Furthermore, EMR plays a role in the ablation of dysplastic or neoplastic tissue, leading to local, curative therapy, as the frequency of synchronous cancers undetected by endoscopy and biopsy in patients with LSBE is about $50 \%$.

\section{Biomarkers}

Biomarkers would play a primary role in the selection of patients with $\mathrm{BE}$ at high risk for progression to HGD or cancer, making surveillance protocols more effective. Currently, no single molecular marker is sufficient to enable prediction for the development of cancer, since the carcinogenesis is a multistep process resulting from alterations in many different genes, categorized according to cellular abnormalities in growth signals reception, reduction or absence of apoptosis, unlimited replication, angiogenesis, and increased invasion and proliferation processes.

Flow cytometry (tetraploidy, aneuploidy), molecular assessment of p16 (methylation, mutation, and loss of heterozygosity $(\mathrm{LOH})$ ), and p53 (mutation, $\mathrm{LOH}$ ) have been evaluated in prospective studies using cancer as an outcome. In the largest study, flow cytometry identified lowand high-risk groups with 0 and $28 \% 5$-year cumulative incidences of cancer, respectively [30]. A recent case-control study reported that nearly two-thirds of cancers arose in patients whose $\mathrm{p} 53$ immunostaining was negative [31]; moreover, progressive reduction in expression of $\mathrm{BCl}-2$ (apoptosis regulator gene) has been recently advocated as a good predictor of progression from LGD to EA [32]. 
Fluorescence in situ hybridization (FISH) is a rapid technique that uses fluorescent molecules as probes to specific DNA sequences and offers many advantages as a method to detect biomarkers of increased risk and particularly helpful for identifying chromosomal abnormalities (aneusomy) and gene deletion, amplification, and translocation. Several studies have shown that a variety of genomic gains and losses precede the development of invasive cancer in BE. Walch et al. [33] described amplification of both HER-2 and 20q13.2 by FISH in a group of patients with EA and reported a progressive increasing rate in the deletion of chromosome Y, p16, and p53 in the $\mathrm{BE}$ cancer sequence. To establish the clinical utility of FISH analysis on cytology specimens, further evaluation in patients with $\mathrm{BE}$, with and without dysplasia, is warranted to determine whether FISH abnormalities are detectable in advance of the development of adenocarcinoma. FISH analysis of cytologic specimens from patients with non-dysplastic $\mathrm{BE}$ and $\mathrm{BE}$ cancer demonstrated a difference in the frequency of 20q13.2 and HER-2 gene amplification in patients with EA compared with those from patients without dysplasia or carcinoma [34]. In 50 bioptic specimens, we found a positive correlation between histopathologic characteristics and HER-2/neu gene expression/amplification along the sequence $\mathrm{BE}$ (21 cases), LGD (12 cases), HGD (13 cases) and cancers (13 cases). Her-2/neu amplification or overexpression was never observed in $\mathrm{BE}$, whereas gene amplification and overexpression was observed in 7 out of 13 adenocarcinomas; therefore, HER-2/neu amplification/overexpression may be considered as a marker of progression from $\mathrm{BE}$ to dysplasia and the FISH technique may represent an important and useful diagnostic tool to integrate the result of HercepTest for selecting patients for immunotherapy [35]. Moreover, after EMR for dysplastic BE, FISH for chromosomes X, Y, 4, 8, 17, showed an increased aneusomy for chromosomes 4, 8, 17, 18 along the sequence of cancer progression with a trend for chromosome 8 to be below the FISH cutoff, and $50 \%$ of cases only showed aneusomy for chromosome 18. Conversely aneusomy was increased for chromosomes 4 and 17, but to a similar extent for LGD and HGD. The presence of the Y chromosome was revealed by FISH in BE and LGD specimens, but not in HGD and EA. FISH is a reliable diagnostic tool to confirm histopathologic diagnosis of HGD. Loss of $Y$ chromosome may be a selective marker of HGD, a finding that warrants further prospective studies with Barrett's associated pre-neoplastic lesions [36].

\section{Conclusions}

The role of screening and surveillance in BE remains controversial, but a tailored screening program would be able to identify high-risk patients to enroll in surveillance programs. Even it is unclear whether proposed international guidelines are cost-effective or affect the outcome, risk stratification of subjects with $\mathrm{BE}$ is the key point to achieve the better results in $\mathrm{BE}$ management and this strategy seems to be the only way to detect early cancer. Technological improvements in endoscopic diagnosis achieved good results in the detection of early abnormalities arising in $\mathrm{BE}$ epithelium, leading to targeted biopsies; moreover, EMR techniques are currently considered the best method for accurate staging. Biomarkers applied for histological analysis could be an adjunctive tool to assess the probability for cancer development leading to enroll patients into surveillance in order to ameliorate the survival from EA.

\section{References}

1 Conio M, Blanchi S, Lapertosa G, et al: Longterm endoscopic surveillance of patients with Barrett's esophagus. Incidence of dysplasia and adenocarcinoma. A prospective study. Am J Gastroenterol 2003;98:19311939.

$\checkmark 2$ Eloubeidi MA, Mason AC, Desmon RA, ElSerag HB: Temporal trends (1937-1997) in survival of patients with esophageal adenocarcinoma in USA: a glimmer of hope? Am J Gastroenterol 2003;98:1627-1633.
-3 Sharma P, McQuaid K, Dent J, et al: A critical review of the diagnosis and management of Barrett's esophagus: the AGA Chicago Workshop. Gastroenterology 2004;127:310330.

4 Gerson LB, Shetler K, Triadafilopoulos G: Prevalence of Barrett's esophagus in asymptomatic individuals. Gastroenterology 2002; 123:461-467.

5 Jankowski JA, Harrison RF, Perry I, Balkwill F, Tselepis C: Barrett's metaplasia. Lancet 2000;356:2079-2085.
-6 Sampliner RE, Garewal HS, Fennerty MB, Aickin M: Lack of impact of therapy on extent of Barrett's esophagus in 67 patients. Dig Dis Sci 1990;35:93-96.

-7 Lagergren J, Bergstrom R, Lindgren A, Nyren O: Symptomatic gastroesophageal reflux as a risk factor for esophageal adenocarcinoma. N Engl J Med 1999;340:825-831.

-8 Inadomi JM, Sampliner R, Lagergren J, Lieberman D, Fendrick M, Vakil N: Screening and surveillance for Barrett's esophagus in high-risk groups: a cost-utility analysis. Ann Intern Med 2003;138:176-186. 
9 Van Sandick JW, Van Lanschot JJ, Kuiken BW, et al: Impact of endoscopic biopsy surveillance of Barrett's esophagus on pathological stage and clinical outcome of Barrett's carcinoma. Gut 1998;43:216-222.

10 Corley DA, Levin TR, Habel LA: Surveillance and survival in Barrett's adenocarcinomas: a population-based study. Gastroenterology 2002;122:633-640.

- 11 Sampliner RE: Practice Parameters Committee ACG: updated guidelines for the diagnosis, surveillance and therapy of Barrett's esophagus. Am J Gastroenterol 2002;97: 1888-1895.

12 Reid BJ, Weinstein WM, Lewin KJ, et al: Endoscopic biopsy can detect high-grade dysplasia or early adenocarcinoma in Barrett's esophagus without grossly recognizable neoplastic lesions. Gastroenterology 1988; 94:81-90.

13 Ormsby AH, Petras RE, Henricks WH, et al: Observer variations in the diagnosis of superficial esophageal adenocarcinoma. Gut 2002;51:671-676.

14 Buttar NS, Wang KK, Sebo TJ, et al: Extent of high-grade dysplasia in Barrett's esophagus correlates with risk of adenocarcinoma. Gastroenterology 2001;120:1630-1639.

15 Buttar NJ, Wang KK, Burgart LJ, et al: Endoscopic appearance and extent of high-grade dysplasia in Barrett's esophagus can predict the presence of cancer at esophagectomy. Am J Gastroenterol 2003;98(suppl):A81.

- 16 Schnell TG, Sontag SJ, Chejfec G, Aranha G, Metz A, O'Connell S, Seidel UJ, Sonnenberg A: Long-term nonsurgical management of Barrett's esophagus with high-grade dysplasia. Gastroenterology 2001;120:1607-1619.

$\checkmark 17$ Sontag SJ: Esophagectomy is the treatment of choice for high-grade dysplasia in Barrett's esophagus. Am J Gastroenterol 2006;101: 2177-2184.

18 Hirota WK, Loughney TM, Lazas DJ, et al: Specialized intestinal metaplasia, dysplasia and cancer of the esophagus and esophagogastric junction: prevalence and clinical data. Gastroenterology 1999;116:277-285.
19 Weston AP, Sharma P, Mathur S, Banerjee S, Jafri AK, Cherian R, McGregor D, Hassanein RS, Hall M: Risk stratification of Barrett's esophagus: updated prospective multivariate analysis. Am J Gastroenterol 2004;99: 1657-1666.

20 Dulai GS, Shekelle PG, Jensen DM, et al: Dysplasia and risk of further neoplastic progression in a Regional Veterans Administration Barrett's Cohort. Am J Gastroenterol 2005; 100:775-783.

21 The Paris Endoscopic Classification of Superficial Neoplastic Lesions: Esophagus, stomach and colon: November 30 to December 1 2002. Gastrointest Endosc 2003;58(6 suppl):S3-S43.

22 Kiesslich R, Hahn M, Herrmann G, Jung M: Screening for specialized columnar epithelium with methylene blue: chromoendoscopy in patients with Barrett's esophagus and a normal control group. Gastrointest Endosc 2001; $53: 47-52$.

-23 Sharma P, Marcon N, Wani S, Bansal A, Mathur S, Sampliner R, Lightdale C: Nonbiopsy detection of intestinal metaplasia and dysplasia in Barrett's esophagus: a prospective multicenter study. Endoscopy 2006;38: 1206-1212.

24 Gono K, Obi T, Yamaguchi M, et al: Appearance of enhanced tissue features in narrowband endoscopic imaging. J Biomed Opt 2004;9:568-577.

25 Yoshida T, Inoue H, Usui S, Satodate H, Fukami N, Kudo SE: Narrow-band imaging system with magnifying endoscopy for superficial esophageal lesions. Gastrointest Endosc 2004;59:288-295.

26 Fock KM, Teo EK, Chan YH, et al: The utility of narrow band imaging in the detection of goblet metaplasia in patients with gastroesophageal reflux disease (abstract). Gastrointest Endosc 2006;63:AB109.

27 Yao K, Takaki Y, Ohara J, et al: Magnification endoscopy outlines the microvascular architecture and extent of Barrett's intramucosal carcinoma prior to endoscopic resection. Gastrointest Endosc 2006;63:10641065 .

28 Saeian K, Staff DM, Vasilopoulos S, Townsend WF, Almagro UA, Komorowski RA, Choi H, Shaker R: Unsedated transnasal endoscopy accurately detects Barrett's metaplasia and dysplasia. Gastrointest Endosc 2002;56:472-478.
29 Ell C, May A, Gossner L, Pech O, Günter E, Mayer G, et al: Endoscopic mucosal resection of early cancer and high-grade dysplasia in Barrett's esophagus. Gastroenterology 2000;118:670-677.

- 30 Teodori L, Gohde W, Persiani M, Ferrario F, Tirindelli Danesi D, Scarpignato C, Di Tondo U, Alo P, Capurso L: DNA/protein flow cytometry as a predictive marker of malignancy in dysplasia-free Barrett's esophagus: 13-year follow-up study on a cohort of patients. Cytometry 1998;34:257-263.

-31 Coggi G, Bosari S, Roncalli M, Graziani D, Bossi P, Viale G, Buffa R, Ferrero S, Piazza M, Blandamura S, Segalin A, Bonavina L, Peracchia A: p53 protein accumulation and p53 gene mutation in esophageal carcinoma. A molecular and immunohistochemical study with clinicopathologic correlations. Cancer 1997;79:425-432.

32 Bani-Hani K, Martin IG, Hardie LF, Mapstone N, Briggs JA, Forman D, Wild CP: Prospective study of cyclin D1 overexpression in Barrett's esophagus: associating with increased risk of adenocarcinoma. J Natl Cancer Inst 2000;92:1316-1321.

-33 Walch A, Specht J, et al: Her-2/neu gene amplification, elevated mRNA expression and protein overexpression in metaplasia-dysplasia-adenocarcinoma in Barrett's esophagus. Lab Invest 2001;81:791-801.

34 Falk GW, Skacel M, Gramlich TL, Casey G, Goldblum JR, et al: Fluorescence in situ hybridization of cytologic specimens from Barrett's esophagus: a pilot feasibility study. Gastrointest Endosc 2004;60:280-284.

35 Rossi E, Villanacci V, Della Casa D, Missale G, Ghedi M, Cengia G, Cestari R: Her-2/neu in Barrett's esophagus: a comparative study between histology, immunohistochemistry, and fluorescence in situ hybridization. Diagn Mol Pathol 2006;25:125-130.

36 Cestari R, Rossi E, Villanacci V, Della Casa D, Missale G, Conio M, Grigolato PG: Dysplasia in Barrett's esophagus FISH: a new approach. Gastroenterology 2005; 128:A240. 